\title{
Antiviral RNAi therapy: emerging approaches for hitting a moving target
}

\author{
JN Leonard and DV Schaffer \\ Department of Chemical Engineering and the Helen Wills Neuroscience Institute, University of California, Berkeley, CA, USA
}

The field of directed RNA interference (RNAi) has rapidly
developed into a highly promising approach for specifically
downregulating genes to alleviate disease pathology. This
technology is especially well-suited to treating viral infec-
tions, and numerous examples now illustrate that a wide
range of viruses can be inhibited with RNAi, both in vitro and
in vivo. One principle that has arisen from this work is that
antiviral RNAi therapies must be tailored to the unique life
cycle of each pathogen, including the choice of delivery
vehicle, route of administration, gene(s) targeted and
regulation and duration of RNAi induction. Although effective
strategies will be customized to each virus, all such therapies
must overcome similar challenges. Importantly, treatment strategies must compensate for the inevitable fact that viral genome sequences evolve extremely rapidly, and computational and bioinformatics approaches may aid in the development of therapies that resist viral escape. Furthermore, all RNAi strategies involve the delivery of nucleic acids to target cells, and all will therefore benefit from the development of enhanced gene design and delivery technologies. Here, we review the substantial progress that has been made towards identifying effective antiviral RNAi targets and discuss strategies for translating these findings into effective clinical therapies.

Gene Therapy (2006) 13, 532-540. doi:10.1038/sj.gt.3302645; published online 22 September 2005

Keywords: RNAi; RNA interference; siRNA; antiviral; resistance; escape

\section{Introduction}

RNA interference (RNAi) is a mechanism for silencing gene expression that has been conserved through evolution in eukaryotes ranging from plants to humans. ${ }^{1}$ In this process, the cellular complex Dicer cleaves a double-stranded RNA (dsRNA) molecule to yield double-stranded duplexes 21-25 nucleotides in length. These short interfering RNAs (siRNA) then guide the RNAiinduced silencing complex (RISC) to cleave target mRNAs that share sequence identity with the siRNA. ${ }^{1-4}$ Since it was first demonstrated that adding exogenous, synthetic siRNA molecules to mammalian cells can induce $\mathrm{RNAi}^{5}$ there have been rapidly expanding efforts to develop RNAi therapies that induce the degradation of target messenger RNA (mRNA) involved in genetically inherited (i.e. dominant) or acquired disorders. This review will focus on the features and challenges unique to applying RNAi towards one important class of acquired diseases: viral infections.

RNAi is well suited as an antiviral therapy for numerous reasons. Antiviral therapeutics hinge upon the ability to discriminate virus from host; however, viruses rely extensively upon the host cells for many functions and activities involved in viral replication and therefore typically offer a very limited number of therapeutic targets. For example, the majority of anti-

Correspondence: DV Schaffer or JN Leonard, 201 Gilman Hall, Berkeley, CA 94720-1462, USA.

E-mail: schaffer@cchem.berkeley.edu or leonardo@berkeley.edu Received 8 July 2005; revised 12 August 2005; accepted 12 August 2005; published online 22 September 2005
HIV small molecule drugs used in highly active antiretroviral therapies (HAART), focus on one of two viral enzymes, reverse transcriptase and protease. ${ }^{6}$ By contrast, because RNAi targets a short stretch of viral nucleic acids rather than a functional domain of a viral protein, even a small viral genome offers a large number of potential targets. Furthermore, in principle the development of RNAi therapeutics can begin once a viral genome sequence is known, whereas the development of small molecule therapies that inhibit complex protein activities requires the prior elucidation of much more biological and functional detail. Finally, although there is limited evidence that RNAi can play a natural antiviral role in mammalian cells, ${ }^{7-10}$ it is clear that it serves as an important defense against viruses in plants and transposable elements in Caenorhabditis elegans. ${ }^{1,11}$ Therefore, human viruses may prove a particularly fruitful therapeutic target for this evolutionarily conserved mechanism.

Along with these potentially advantageous features, viruses pose a number of unique challenges for RNAibased therapies. Viruses replicate extremely rapidly and accumulate mutations and short deletions, particularly viruses with life cycles that involve RNA genomes. As discussed in further detail below, since RNAi relies upon nearly perfect sequence identity between an siRNA guide and the mRNA target, the accumulation of mutations can render a virus resistant to RNAi suppression, analogous to the evolution of viral resistance to HAART. ${ }^{6}$ Furthermore, during viral replication, dsRNA encoding viral proteins or the full genome is often generated, potentially exposing the virus to host cell RNAi. As a result, it is not surprising that numerous 
viruses have evolved efficient suppressors of RNAi. Although this phenomenon is better characterized in plant infections than in animal viruses, ${ }^{1}$ the apparent presence of RNAi suppressors in human viruses indicates that they could impact antiviral RNAi strategies. 7,8,10,12 For instance, the vaccinia E3L and influenza NS1 proteins suppress RNAi when expressed in heterologous systems, ${ }^{12}$ although it is not yet clear whether this activity was 'intentionally' evolved by the virus or is a side effect of the ability of these proteins to bind dsRNA. Furthermore, it was recently reported that the human immunodeficiency virus type 1 (HIV-1) transcriptional activator protein (Tat) also suppresses RNAi by directly inhibiting Dicer. ${ }^{10}$ Targeting an early stage in the viral life cycle before a suppressor accumulates, or directly targeting the suppressor mRNA, may be necessary to circumvent these mechanisms of viral resistance.

\section{The delivery challenge}

In general, the RNAi-inducing cargo and its delivery system should be designed to match the life cycle and pathology of the target virus. The first option is to deliver synthetic siRNA molecules, either naked or complexed with a synthetic vector. ${ }^{5,13,14}$ Alternatively, one can deliver plasmid or viral vector constructs that induce cells to transcribe two complementary RNA molecules that will hybridize to generate an siRNA, or a short hairpin RNA (shRNA) that folds to yield a Dicer substrate. ${ }^{13,15,16}$ Synthetic siRNAs can provide rapid but transient suppression, which is appropriate for acute viral infections such as influenza or SARS, ${ }^{14,17-20}$ whereas delivery of expression constructs can provide more sustained RNAi that is suitable for chronic infectious such as hepatitis or HIV-1. ${ }^{10,16,21-33}$ Likewise, delivery should be focused to tissues where the target pathogen resides and replicates, somewhat analogous to targeting vaccine delivery to focus immunity in tissues that lie in the pathogen's infectious route of entry into an organism. ${ }^{34}$ For example, influenza and respiratory syncytial virus $(\mathrm{RSV})^{35}$ therapies are most appropriately delivered by inhalation, ${ }^{18}$ whereas hepatitis therapy can be administered intravenously for liver targeting. ${ }^{13,22}$ Similarly, $\mathrm{T}$ cells and macrophages can be protected against HIV by harvesting and transducing hematopoietic stem cells with a virus ex vivo to introduce anti-HIV RNAi constructs. $^{28}$

In addition, the RNAi-inducing nucleic acids must be delivered within a therapeutic window of dosage, as dose-dependence has been observed both in vitro and in vivo. Lentiviral vector delivery of an shRNA-expressing construct progressively knocked down target gene expression in single cells with increasing viral dosage. ${ }^{36}$ Likewise, in a study of intranasal delivery of polyplex delivery vehicles composed of synthetic anti-influenza siRNAs and the polycationic polymer polyethylenimine, higher siRNA doses protected mice challenged with influenza more effectively. ${ }^{14}$ Efficient nucleic acid delivery has been a major challenge in the parallel field of gene therapy, so all delivery vehicles and routes of administration for RNAi-inducing cargo will benefit from prior and continual progress in synthetic and viral vector development. ${ }^{37}$
In addition to a lower delivery limit for efficacy, several phenomena restrict the maximal dosage of RNAi that a host can tolerate. Long dsRNAs, the natural substrates that Dicer processes to generate siRNAs, are known to activate protein kinase $R$ and thereby induce interferon expression, whereas siRNAs themselves are not typically associated with an innate immune response. However, high dosages of synthetic siRNA delivery can stimulate cytotoxic interferon and inflammatory cytokine secretion in a sequence-dependent fashion. ${ }^{38}$ In addition, while siRNAs target degradation of mRNAs with a high degree of sequence specificity, they can in some cases also eliminate mRNA containing similar sequences, leading to broader, unintended modulation of host gene expression. $^{39,40}$ These nonspecific or off-target effects lead to the possibility of clinical side effects. Furthermore, shRNAs interact with another endogenous cellular pathway for suppression of gene expression, the microRNA (miRNA) system. miRNAs are initially transcribed as partially double-stranded precursors and are subsequently processed by the RNase III enzyme Drosha to yield RNA hairpins. These hairpins are then exported from the nucleus by Exportin 5 and subsequently suppress the cytoplasmic translation of target mRNAs. Exogenous shRNAs expressed in the nucleus after therapeutic gene delivery also undergo Exportin 5dependent nuclear export, which can saturate this transport mechanism. ${ }^{41} \mathrm{~A}$ recent report demonstrated that high-efficiency delivery of certain antihepatitis shRNA expression constructs to the murine liver using vectors based on adeno-associated virus serotype 8 could induce significant liver toxicity, likely through competitive inhibition of endogenous miRNA mechanisms. ${ }^{42}$ Finally, the RNAi pathway consumes significant amounts of ATP, so constitutive expression of siRNA or shRNA constructs could metabolically tax host cells. ${ }^{43}$

One approach to overcome such side effects is to limit RNAi to those cells infected with the viral pathogen. The creation of shRNA expression constructs driven by RNA polymerase II promoters ${ }^{44}$ has enabled the development of regulated promoters activated by viral infection. For example, in a recent study an antihepatitis $\mathrm{C}$ virus (HCV) siRNA construct was inserted in front of the HIV LTR promoter, which contains two NF- $\kappa \mathrm{B}$ response elements and is thereby activated by HCV infection. The delivery of this construct to HepG2 cells transfected with fulllength hepatitis $C$ virus cDNA significantly silenced viral gene expression. ${ }^{24}$ Similarly, Unwalla et al. ${ }^{30}$ described a Tat-inducible vector that conditionally expresses antiHIV shRNA only when HIV is present, thereby inhibiting HIV via a negative feedback mechanism. Alternatively, synthetic siRNA can be administered only upon viral infection, and the development of targeted delivery systems may enable specific delivery to affected cell types. ${ }^{45}$

Despite the challenges, a large and growing body of work demonstrates the far-reaching promise of antiviral RNAi once these obstacles can be fully overcome.

\section{Viral target choice}

RNAi has been used to inhibit the expression and block the replication of a broad range of viral species from many families of human and mammalian pathogens 
Table 1 Viruses inhibited with RNAi

\begin{tabular}{|c|c|c|c|c|c|c|c|}
\hline Virus & Family & Genome & RNAi target & Type & System (cell line/other) & $\begin{array}{l}\text { RNAi } \\
\text { inducer }\end{array}$ & References \\
\hline EBV & Herpesviridae & dsDNA & Zta & $\mathrm{v}$ & NA, 293 & shRNA (p) & 46 \\
\hline FMDV & Picornoviridae & RNA (+) & VP1 & $\mathrm{v}$ & bhk-21, suckling mice & shRNA $(p)$ & 47 \\
\hline FMDV & Picornoviridae & RNA (+) & $3 \mathrm{P}, 3 \mathrm{D}$ & $\mathrm{v}$ & bhk-21 & siRNA & 48 \\
\hline HBV & Hepadnaviridae & $\mathrm{dsDNA}^{\mathrm{c}}$ & HBsAg & $\mathrm{v}$ & HepG2.2.15, mice & siRNA & 21 \\
\hline HBV & Hepadnaviridae & $\mathrm{dsDNA}^{\mathrm{c}}$ & core, Pol, S1, X & $\mathrm{v}$ & $\mathrm{HuH}-7$, mice & shRNA (p) & 22 \\
\hline HBV & Hepadnaviridae & $\mathrm{dsDNA}^{\mathrm{c}}$ & $\mathrm{X}, \mathrm{S}$ & $\mathrm{v}$ & $\mathrm{HuH}-7$, mice & shRNA $(p)$ & 23 \\
\hline $\mathrm{HCV}$ & Flaviviridae & RNA (+) & NS3, NS5B & $\mathrm{v}$ & $\mathrm{HuH}-7$ & siRNA & 49 \\
\hline $\mathrm{HCV}$ & Flaviviridae & RNA (+) & $5^{\prime}$ NTR, core & $\mathrm{v}$ & HuH-7 & siRNA & 50 \\
\hline $\mathrm{HCV}$ & Flaviviridae & RNA (+) & $\mathrm{C}$ & $\mathrm{v}$ & HepG2 & $\begin{array}{l}\text { shRNA } \\
(\text { sv } 40)\end{array}$ & 24 \\
\hline $\mathrm{HCV}$ & Flaviviridae & RNA (+) & NS5B & $\mathrm{v}$ & HuH-7 & siRNA & 51 \\
\hline $\mathrm{HCV}$ & Flaviviridae & RNA (+) & La, PTB, hVAP-33 & c & $\mathrm{HuH}-7$ & $\begin{array}{l}\text { shRNA (p, } \\
\text { ad) }\end{array}$ & 75 \\
\hline HIV & Retroviridae & RNA (+) & CXCR4, CCR5 CD4 & c & Magi-CXCR4+,-CCR5+, PBMCs & $\operatorname{shRNA}(p)$ & 69 \\
\hline HIV & Retroviridae & RNA (+) & $\operatorname{Rev}$ & $\mathrm{v}$ & CD34+ HSC, scid mice & shRNA (l) & 16 \\
\hline HIV & Retroviridae & RNA (+) & $\mathrm{Gag}$ & $\mathrm{v}$ & Jurkat, CEM-SS, HeLa-CD4+ & $\operatorname{shRNA}(p)$ & 10 \\
\hline HIV & Retroviridae & RNA (+) & Tat, Rev & $\mathrm{v}$ & H9 & shRNA (aav) & 27 \\
\hline HIV & Retroviridae & RNA (+) & CусT1, CDK9 & c & HeLa & siRNA & 72 \\
\hline HIV & Retroviridae & RNA (+) & Nef & $\mathrm{v}$ & SupT1 & shRNA (r) & 29 \\
\hline HIV & Retroviridae & RNA (+) & LTR-TAR, Vir, Nef & $\mathrm{v}$ & HeLa-CD4+, PBL & siRNA (p) & 25 \\
\hline HIV & Retroviridae & RNA (+) & CXCR4 & $\mathrm{c}$ & HeLa-CD4+/CXCR4+ & siRNA & 68 \\
\hline HIV & Retroviridae & RNA (+) & PARP-1 & c & HeLa, J111 & siRNA & 71 \\
\hline HIV & Retroviridae & RNA (+) & Arp2 & c & 293 & shRNA $(p)$ & 32 \\
\hline HIV & Retroviridae & RNA (+) & Tat,Rev & $\mathrm{v}$ & $293 / \mathrm{EcR}$ & $\operatorname{siRNA}(\mathrm{p})$ & 26 \\
\hline HIV & Retroviridae & RNA (+) & Tat, Rev & $\mathrm{v}$ & PBMCs, CD34+ monocytes & shRNA (1) & 28 \\
\hline HIV & Retroviridae & RNA (+) & CXCR4, CCR5 & $\mathrm{c}$ & U87-CD4-CXCR4, -CCR5 & siRNA & 67 \\
\hline HIV & Retroviridae & RNA (+) & Sam68 & c & 293T, HeLa & shRNA (p) & 33 \\
\hline HIV & Retroviridae & RNA (+) & CD4, Gag & $c, \mathrm{v}$ & $\begin{array}{l}\text { Magi-CCR5, HeLa-CD4, H9, } \\
\text { ACH-2, PBL }\end{array}$ & siRNA & 52 \\
\hline HIV & Retroviridae & RNA (+) & Rev & $\mathrm{v}$ & 293/EcR, CEM, CD34+ HSC & $\operatorname{shRNA}(p, 1)$ & 30 \\
\hline HIV & Retroviridae & RNA (+) & Nef & $\mathrm{v}$ & SupT1 & shRNA $(\mathrm{r})$ & 31 \\
\hline HIV & Retroviridae & RNA (+) & hRIP & c & $\begin{array}{l}\text { HeLa, HL2/3, Jurkat, primary } \\
\text { macrophages }\end{array}$ & siRNA & 73 \\
\hline HTLV & Retroviridae & RNA (+) & TORC3 & c & $293 \mathrm{~T}$ & siRNA & 74 \\
\hline HPV & Papovaviridae & dsDNA & E6 & $\mathrm{v}$ & $\begin{array}{l}\text { HeLa, SiHa, MCF-7, MeWo, } \\
\text { H1299 }\end{array}$ & $\begin{array}{l}\text { siRNA, } \\
\text { shRNA }(p)\end{array}$ & 54 \\
\hline HPV & Papovaviridae & dsDNA & E6, E7 & $\mathrm{v}$ & HeLa & siRNA & 53 \\
\hline IVA & Orthomyxoviridae & RNA (-) & $\mathrm{NP}, \mathrm{PA}$ & $\mathrm{v}$ & MDCK, Vero, chicken embryos & siRNA & 17 \\
\hline IVA & Orthomyxoviridae & RNA (-) & NP, PA, PB1 & $\mathrm{v}$ & MDCK, Vero, mice & $\begin{array}{l}\text { siRNA, } \\
\text { shRNA }(p, 1)\end{array}$ & 14 \\
\hline IVA & Orthomyxoviridae & RNA (-) & M2 splice junction & $\mathrm{v}$ & 293T, BHK & shRNA (p) & 55 \\
\hline IVA & Orthomyxoviridae & RNA (-) & NP, PA & $\mathrm{v}$ & MDCK, mice & siRNA & 18 \\
\hline Marburg & Filoviridae & RNA (-) & NP, VP35 and VP30 & $\mathrm{v}$ & Vero, HeLa-CCL-2 & siRNA & 56 \\
\hline PIV & Paramyxoviridae & RNA (-) & F, HN, RdRP & $\mathrm{v}$ & A549 & siRNA & 57 \\
\hline PIV & Paramyxoviridae & RNA (-) & $\mathrm{P}$ & $\mathrm{v}$ & mice & siRNA & 35 \\
\hline Poliovirus & Picornoviridae & RNA (+) & capsid, P3 & $\mathrm{v}$ & $\begin{array}{l}\text { HeLa S3, mouse embryonic } \\
\text { fibroblasts }\end{array}$ & siRNA & 58 \\
\hline Poliovirus & Picornoviridae & RNA (+) & capsid, P3 & $\mathrm{v}$ & HeLa S3, P19 & $\begin{array}{l}\text { siRNA, } \\
\text { esiRNA }\end{array}$ & 59 \\
\hline Rotavirus & Reoviridae & dsRNA & VP4, VP7 & $\mathrm{v}$ & MA104 & siRNA & 61 \\
\hline Rotavirus & Reoviridae & dsRNA & VP4 & $\mathrm{v}$ & MA104 & siRNA & 60 \\
\hline RSV & Paramyxoviridae & RNA (-) & $\mathrm{P}, \mathrm{F}, \mathrm{SH}$ & $\mathrm{v}$ & A549 & siRNA & 57 \\
\hline RSV & Paramyxoviridae & RNA (-) & $\mathrm{P}, \mathrm{F}$ & $\mathrm{v}$ & A549 & siRNA & 62 \\
\hline RSV & Paramyxoviridae & RNA (-) & $\mathrm{P}$ & $\mathrm{v}$ & A549, mice & siRNA & 35 \\
\hline SARS-CoV & Coronaviridae & RNA (+) & $\operatorname{Rep} 1 \mathrm{~A}$ & $\mathrm{v}$ & FRhk-4 & siRNA & 19 \\
\hline SARS-CoV & Coronaviridae & RNA (+) & Leader & $\mathrm{v}$ & 293T, Vero E6 & $\begin{array}{l}\text { siRNA, } \\
\text { shRNA (p) }\end{array}$ & 63 \\
\hline SARS-CoV & Coronaviridae & RNA (+) & $\mathrm{N}$ & $\mathrm{v}$ & Vero E6 & $\operatorname{shRNA}(p)$ & 64 \\
\hline SARS-CoV & Coronaviridae & RNA (+) & 48 genomic \& coding & $\mathrm{v}$ & FRhk-4 & siRNA & 20 \\
\hline VSV & Rhabdoviridae & RNA (-) & $\mathrm{M}, \mathrm{RdRP}$ & $\mathrm{v}$ & HEp-2 & siRNA & 57 \\
\hline WNV & Flaviviridae & RNA (+) & capsid, NS5 & $\mathrm{v}$ & $293 \mathrm{~T}$ & shRNA (p) & 55 \\
\hline
\end{tabular}

${ }^{\mathrm{a}} \mathrm{v}$ : viral target, c: cellular target.

'Endogenous expression indicated by aav (AAV vector), ad (adenoviral vector), 1 (lentiviral vector), $r$ (retroviral vector) $\mathrm{p}$ (plasmid), sv40 (SV40 vector).

'HBV uses pregenomic RNA to template genomic DNA synthesis. 

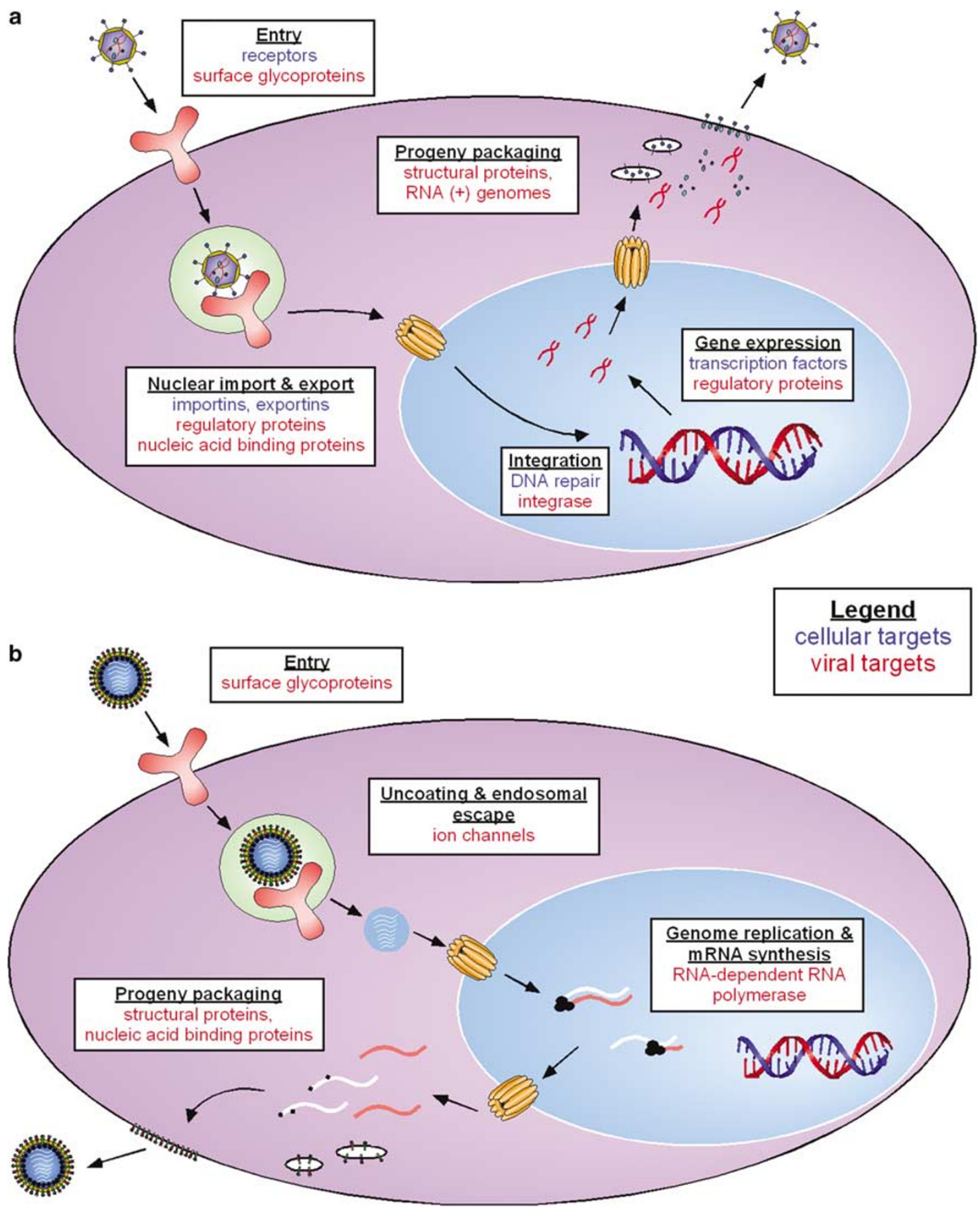

Figure 1 RNAi target selection must be tailored to the unique life cycle of the virus. HIV (a) and influenza (b) both have single-stranded RNA genomes, but each is susceptible to RNAi-mediated blocks at different points in its life cycle. This figure highlights viral and cellular targets that have been successfully utilized against HIV and influenza and indicates the steps of the viral replication cycles at which such blocks would occur. However, it is not intended to exhaustively depict all potential targets. Cellular targets appear in blue text, and viral targets appear in red.

(summarized in Table 1). However, viruses vary widely in their basic biology and reproductive mechanisms, which suggests that unique strategies are needed to inhibit each particular virus (see Figure 1). As RNAi is currently understood, RISC-mediated RNA cleavage is restricted to cytoplasmically located target molecules, and since all viruses hijack cellular translation machinery to express their proteins, the targeting of virally encoded cytoplasmic mRNAs is a common locus at which all viruses should be susceptible to inhibition. In support of this assertion, it has been shown that a broad spectrum of viruses can be inhibited by targeting essential viral mRNAs. To date, the list of viruses successfully inhibited in this manner includes Epstein-Barr virus (EBV), ${ }^{46}$ footand-mouth disease virus (FMDV), ${ }^{47,48}$ hepatitis B virus $(\mathrm{HBV}),{ }^{21-23}$ hepatitis $\mathrm{C}$ virus (HCV), ${ }^{24,49-51}$ human immunodeficiency virus type 1 (HIV-1), 10,16,25-31,52 human papilloma virus $(\mathrm{HPV}),{ }^{53,54}$ influenza virus $\mathrm{A}$ 
(IVA), ${ }^{14,17,18,55}$, Marburg virus, ${ }^{56}$ human parainfluenza virus (PIV), ${ }^{35,57}$ poliovirus, ${ }^{58,59}$ rotavirus, ${ }^{60,61} \mathrm{RSV}^{35,57,62}$ severe acute respiratory syndrome - associated coronavirus (SARS-CoV), ${ }^{19,20,63,64}$ vesicular stomatitis virus (VSV), ${ }^{57}$ and West Nile virus (WNV). ${ }^{55}$ In most of these cases, inhibition was achieved by directly reducing the viruses' ability to reproduce. However, Butz et al. ${ }^{54}$ demonstrated that HPV pathogenesis could be reduced by targeting the viral inhibitor of apoptosis, E6, to limit viral spread by selectively inducing apoptosis in HPV-infected cells. This example also illustrates that each type of viral infection offers unique treatment options.

When tailoring an RNAi-based therapy to a particular virus, the most important consideration is the unique life cycle of that virus. To a first degree of approximation, these life cycles can be grouped by the type of nucleic acids of which the viral genome is composed. For viruses with DNA genomes, the sole option is to target viral mRNA. For example, targeting HPV mRNAs was sufficient to block viral replication and reduce pathogenesis. ${ }^{53,54}$ However, when targeting viruses with positivestrand RNA genomes (where the viral genomes are transcribed in the same orientation as the viral mRNAs), genomic RNA and mRNA might be degraded simultaneously due to the fact that they share sequence identity. Direct cleavage of positive-strand genomic RNA has been demonstrated for $\mathrm{HIV}^{25}$ and HBV's pregenomic RNA (which serves as both an mRNA and a template for reverse transcription of genomic DNA) appears to be similarly susceptible, ${ }^{21-23}$ but it is not yet clear whether simultaneous degradation of genomic and mRNA underlies the observed viral inhibition of the positivestrand RNA viruses HCV ${ }^{24,49-51}$ and SARS-CoV. ${ }^{19,20,63,64}$

The situation appears more complex when targeting viruses with negative-strand RNA genomes. For example, it was shown when applying anti-influenza virus siRNAs that could potentially induce RNAi against either sense RNA (mRNA and genome-complementary RNA made during replication) or antisense RNA (genomic RNA), only mRNA was susceptible to RISCmediated cleavage. ${ }^{17}$ This selectivity was potentially due partially to the fact that influenza viral replication takes place in the nucleus, which isolates and protects viral RNA from RISC. However, a similar differential susceptibility of viral mRNA and genomic RNA was also observed when targeting RSV, which has a negativestrand RNA genome, ${ }^{62}$ and rotavirus, which has a dsRNA genome. ${ }^{61}$ These latter viruses reproduce in the cytoplasm and should thus be susceptible to RISC; however, again only viral mRNA was degraded. This selective vulnerability can be at least partly explained by the fact that the genomic RNAs of cytoplasmically replicating viruses are tightly bound by viral proteins that may protect the RNA from RISC. Moreover, since effective siRNAs are loaded asymmetrically into RISC, with a preference for one strand over the other, it is unlikely that both negative-strand RNA genomes and viral mRNAs will be targeted simultaneously by a single siRNA. ${ }^{65}$ However, synergistic efficacy could be obtained by targeting the RNA-binding proteins expressed by cytoplasmically replicating RNA viruses, since such inhibition could both repress the production of new viruses and render genomic RNA more susceptible to RNAi-mediated cleavage. In any case, it is clear that each unique viral replication mechanism must be taken into consideration to achieve maximal RNAi-mediated inhibition.

\section{Viral evolution}

One drawback to targeting viral genes with RNAi is that viral mutation can lead to a loss of sensitivity to RNAi. This is an especially problematic possibility for the cases of RNA viruses, which accumulate point mutations up to $10^{7}$-fold more rapidly than do their counterparts with DNA genomes. ${ }^{66}$ In illustration of this problem, RNAiresistant mutants arose in cell culture models while targeting $\mathrm{HIV},{ }^{27,29,31}$ poliovirus, ${ }^{58,59} \mathrm{HCV}^{51}$ and $\mathrm{HBV}^{23}$ Although most of these mutants achieved resistance through point mutation or deletion of the target sequence, one HIV mutant escaped RNAi suppression by accumulating mutations outside of the target sequence, thereby creating a new local RNA secondary structure that presumably excluded RISC. ${ }^{31}$ These results demonstrate conclusively that any effective RNAi-based antiviral therapy must compensate for the evolutionary potential of the pathogen.

It is possible that the problem of viral evolution can be circumvented by directing RNAi against essential cellular cofactors. Ideally, the loss of these cellular targets must block viral replication without inducing loss of function pathology in the cells. For example, HIV utilizes the chemokine receptors CCR5 and CXCR4 as co-receptors for cell entry, and downregulation of these genes in immune cells with RNAi blocks HIV infection. ${ }^{67-69}$ Importantly, humans who are homozygous for deletions that abolish CCR5 expression have apparently normal immune function and are naturally more resistant to $\mathrm{HIV}^{70}$ In addition, targeting cellular receptors could truly protect cells from initial viral infection rather than only impair postinfection viral reproduction, which is what targeting viral genes achieves. In addition, producing a block at a viral entry step could strand the virus in the extracellular space for greater amounts of time, rendering it more susceptible to immune surveillance and clearance. Finally, for the virus to compensate for the loss of its primary receptor and/or coreceptor, it would need to make a challenging evolutionary leap comparable to acquiring novel tissue tropism or crossing a species barrier.

In addition to cell surface receptors, a number of host cellular RNAi targets that provide blocks at intracellular steps have also been identified. For example, the Arp2/3 complex mediates the nucleation step in actin polymerization, and this mechanism is used by some viruses and bacteria for intracellular transport. HIV infection was accordingly halted at the step of intracellular trafficking to the nucleus when cellular Arp2 was inhibited. ${ }^{32}$ Downregulation of poly(ADP-ribose) polymerase 1 (PARP-1) abolished HIV integration and interfered with the activation of NF- $\kappa \mathrm{B}$-dependent genes, including the $\mathrm{HIV}$ long terminal repeat (LTR) promoter. ${ }^{71}$ It is also possible to impede viral regulatory functions by targeting cellular proteins that interact with viral regulatory factors after the initiation of viral transcription. For example, HIV gene expression and replication are critically dependent upon the recruitment of the host transcriptional complex pTEFb, composed of cyclin $\mathrm{T} 1$ 
and CDK9, by the viral protein Tat. RNAi targeting of cyclin $\mathrm{T} 1$ and $\mathrm{CDK} 9$ was shown to impair HIV replication. ${ }^{72}$ In addition, severely attenuated viral replication was observed when RNAi was directed against either Sam68 or human Rev-interacting protein (hRIP), both of which are required for HIV Rev-mediated nuclear export of unspliced viral genomic RNA. ${ }^{33,73}$

Analogous to work with HIV, HTLV's Tax-mediated transcriptional activation was blocked by targeting TORC3, an essential cellular factor recruited to the

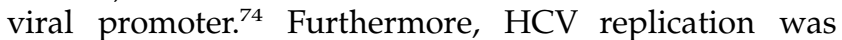
reduced by directing RNAi against various cellular factors, including La, polypyrimidine tract-binding protein (PTB), and human VAMP-associated protein of $33 \mathrm{kDa}$ (hVAP-33). ${ }^{75}$ Despite these positive results, a potential shortcoming of the cellular target approach is suggested by experiences with HIV-1. In general, viral replication was more severely crippled by targeting viral genes than by downregulating cellular genes alone. $^{76}$ Moreover, before any such cellular targetdirected inhibition can be applied as a therapy, for patient safety it must be thoroughly demonstrated that even a total loss of target gene expression would not be harmful. Likewise, this approach in particular could benefit from the further development of strategies to spatially (in cells and tissues for which a virus has tropism) and/or temporally (upon viral infection) regulate RNAi induction.

Another strategy by which viral evolution might be thwarted is the simultaneous application of multiple RNAi-inducing species that target several sequences. This combinatorial strategy is the basis for HAART treatment for HIV, which has improved patient response to antiviral drugs by administering a cocktail of several compounds that targets multiple viral enzymes. ${ }^{77}$ In fact, combination RNAi might improve efficacy over singletarget treatment by several mechanisms. First, it has been shown that a synergistic inhibition of viral replication was achieved by targeting multiple loci of the same gene. ${ }^{68}$ Secondly, by simultaneously targeting several viral genes and thus blocking multiple steps in the viral life cycle, enhanced inhibition can also be achieved..$^{20,48}$ Similarly, simultaneous targeting of multiple cellular cofactors, such as the HIV receptor and coreceptors, led to enhanced viral inhibition. ${ }^{69}$ Finally, to overcome this more potent inhibition, the virus would have to accumulate multiple mutations, which significantly reduces the possibility that a fully resistant mutant will emerge. ${ }^{51,59,78}$ An analogous combinatorial approach is to combine RNAi interference with other gene therapymediated inhibitors of viral replication. For example, Rossi et al. ${ }^{28}$ achieved enhanced HIV inhibition by using a gene therapy vector that simultaneously expressed siRNA directed against the HIV Rev protein, a nucleolarlocalizing TAR decoy RNA, and a ribozyme that downregulates expression of the cellular CCR5 co-receptor. In fact, RNAi might be combined effectively with any number of antiviral therapeutics, including dominantnegative antiviral proteins and more traditional chemotherapeutic drugs, such as those currently used in HAART. Future work will need to synthesize these principles to identify antiviral strategies that provide effective and long-term suppression of viral replication and pathogenesis.

\section{Future directions}

It is clear from the abundance of studies in recent years that many possible strategies and options exist for blocking viral replication with RNAi. Therefore, as the field advances towards clinical trials, it is important to pose a key question: how does one design an optimal antiviral RNAi-based therapy? In addition to the extensive empirical data available and the safety, efficacy, and viral escape considerations described above, it would be useful to draw upon the growing wealth of computational and bioinformatics approaches to identify promising starting points for the empirical testing of antiviral strategies. Viruses often contain complex gene regulation circuits that exhibit nonlinear behavior, ${ }^{79}$ and targeting specific loci in this circuitry may optimally suppress viral replication. For example, in analysis of a bacteriophage T7 model system, Endy et al. ${ }^{80}$ employed a computational model to determine that antisensemediated therapies should target viral genes involved in negative-feedback regulation in order to maximize resistance to viral escape. We recently reported the use of an agent-based stochastic simulation describing HIV replication and evolution in the presence of RNAi directed against the HIV TAR element. This model was used to identify criteria of siRNA dose, delivery efficiency, and RNAi target sequence choice (within TAR) required to maximize the probability of long-term viral inhibition. ${ }^{78}$

In the preparation of clinical antiviral RNAi therapies, it would also be prudent to draw upon the experiences gained using HAART to manage HIV infections. Clinical success with HAART can be improved by analyzing patients' primary viral isolates to predict viral drug susceptibility and thereby design drug regimens that resist treatment failure. ${ }^{77} \mathrm{~A}$ direct, although costly, approach is to perform batteries of in vitro assays to test viral isolate susceptibility to different therapies. However, a more cost-effective approach employs statistical bioinformatics analysis of the viral genotype, that is, the dominant viral genetic sequences present in a patient's isolate. ${ }^{81}$ For the most part, such approaches use large data sets to correlate viral genotypes with past histories of success or failure on specific drug regimens to guide the choice of optimal drugs. Recent developments have enhanced the predictive ability of such bioinformatics methods by explicitly including considerations of viral evolution. For example, Beerenwinkel et al. ${ }^{82}$ have developed a strategy that utilizes the predicted phenotypes of both the viral isolate and its closest genetic 'neighbors'. Although such approaches have proven effective in many cases and at least in the short term, many patients eventually develop drug-resistant infections. ${ }^{83}$ In total, these experiences suggest that computational tools, in combination with relevant in vitro experiments and animal models, can improve the chances for clinical success (and avoid potential pitfalls) as antiviral RNAi therapies are prepared for human trials. Importantly, such proactive measures might preclude the need to amass large databases of clinical trials before effective solutions can be identified.

In summary, since the first demonstration of RNAi in a mammalian cell $<5$ years ago, ${ }^{5}$ there has been enormous progress towards applying RNAi to antiviral therapy. Furthermore, clinical applications will benefit from the 
advantages that RNAi-based antiviral therapies hold over traditional drugs. Since the RNAi mechanism makes use of an innate cellular pathway, requiring the introduction of only a dsRNA trigger, it offers the potential for effective antiviral therapies with minimal side effects. This consideration is especially relevant when compared to current treatments for chronic infections, such as HIV, which consist of chemotherapeutic drugs that require complicated dosing schedules, can induce severe side effects that undermine patient compliance, and can even cause novel medical problems. ${ }^{84}$ Moreover, although each antiviral RNAi therapy will need to target sequences tailored to the particular virus, the actual therapeutic delivery methods will be largely similar across many targets. Consequently, it should be possible to develop new RNAi-based therapies more rapidly than can be achieved with small molecule drugs. Such a readily adaptable approach would be useful in targeting viruses whose dominant genotype and phenotype vary seasonally, such as influenza, especially in cases where vaccination is difficult to predict or in years when the vaccine ineffectively targets the dominant strains. Similarly, once an effective antiviral RNAi-based therapy is developed, it might serve as a basis for quickly developing treatments for emerging viral infections, such as Ebola or SARS-CoV. Such treatments could be made available for treating acute infections, even before the full biology of such novel viruses has been elucidated. Finally, RNAi-based therapies might provide a first line of defense against viral strains that acquire, through natural or human means, resistance to drugs and vaccines previously found to be effective. Although much work remains to be carried out before clinical success is realized, RNAi-based therapies have emerged as highly promising, modular and versatile prospects for treating viral infections.

\section{References}

1 McManus MT. Small RNAs and immunity. Immunity 2004; 21: $747-756$.

2 Fire A, Xu S, Montgomery MK, Kostas SA, Driver SE, Mello CC. Potent and specific genetic interference by double-stranded RNA in Caenorhabditis elegans. Nature 1998; 391: 806-811.

3 Hannon GJ. RNA interference. Nature 2002; 418: 244-251.

4 Sharp PA. RNA interference - 2001. Genes Dev 2001; 15: 485-490.

5 Elbashir SM, Harborth J, Lendeckel W, Yalcin A, Weber K, Tuschl T. Duplexes of 21-nucleotide RNAs mediate RNA interference in cultured mammalian cells. Nature 2001; 411: 494-498.

6 Clavel F, Hance AJ. HIV drug resistance. N Engl J Med 2004; 350: 1023-1035.

$7 \mathrm{Li} \mathrm{H}, \mathrm{Li}$ WX, Ding SW. Induction and suppression of RNA silencing by an animal virus. Science 2002; 296: 1319-1321.

8 Lecellier CH, Dunoyer P, Arar K, Lehmann-Che J, Eyquem S, Himber $\mathrm{C}$ et al. A cellular microRNA mediates antiviral defense in human cells. Science 2005; 308: 557-560.

9 Bucher E, Hemmes H, de Haan P, Goldbach R, Prins M. The influenza A virus NS1 protein binds small interfering RNAs and suppresses RNA silencing in plants. J Gen Virol 2004; 85: 983-991.

10 Bennasser Y, Le SY, Benkirane M, Jeang KT. Evidence that HIV-1 encodes an siRNA and a suppressor of RNA silencing. Immunity 2005; 22: 607-619.

11 Roth BM, Pruss GJ, Vance VB. Plant viral suppressors of RNA silencing. Virus Res 2004; 102: 97-108.
12 Li WX, Li H, Lu R, Li F, Dus M, Atkinson P et al. Interferon antagonist proteins of influenza and vaccinia viruses are suppressors of RNA silencing. Proc Natl Acad Sci USA 2004; 101: 1350-1355.

13 McCaffrey AP, Meuse L, Pham TT, Conklin DS, Hannon GJ, Kay MA. RNA interference in adult mice. Nature 2002; 418: 38-39.

14 Ge Q, Filip L, Bai A, Nguyen T, Eisen HN, Chen J. Inhibition of influenza virus production in virus-infected mice by RNA interference. Proc Natl Acad Sci USA 2004; 101: 8676-8681.

15 Paddison PJ, Caudy AA, Bernstein E, Hannon GJ, Conklin DS. Short hairpin RNAs (shRNAs) induce sequence-specific silencing in mammalian cells. Genes Dev 2002; 16: 948-958.

16 Banerjea A, Li MJ, Bauer G, Remling L, Lee NS, Rossi J et al. Inhibition of HIV-1 by lentiviral vector-transduced siRNAs in T lymphocytes differentiated in SCID-hu mice and CD34+ progenitor cell-derived macrophages. Mol Ther 2003; 8: 62-71.

17 Ge Q, McManus MT, Nguyen T, Shen CH, Sharp PA, Eisen HN et al. RNA interference of influenza virus production by directly targeting mRNA for degradation and indirectly inhibiting all viral RNA transcription. Proc Natl Acad Sci USA 2003; 100: 2718-2723.

18 Tompkins SM, Lo CY, Tumpey TM, Epstein SL. Protection against lethal influenza virus challenge by RNA interference in vivo. Proc Natl Acad Sci USA 2004; 101: 8682-8686.

19 He ML, Zheng B, Peng Y, Peiris JS, Poon LL, Yuen KY et al. Inhibition of SARS-associated coronavirus infection and replication by RNA interference. JAMA 2003; 290: 2665-2666.

20 Zheng BJ, Guan Y, Tang Q, Du C, Xie FY, He ML et al. Prophylactic and therapeutic effects of small interfering RNA targeting SARS-coronavirus. Antivirus Ther 2004; 9: 365-374.

21 Giladi H, Ketzinel-Gilad M, Rivkin L, Felig Y, Nussbaum O, Galun E. Small interfering RNA inhibits hepatitis B virus replication in mice. Mol Ther 2003; 8: 769-776.

22 McCaffrey AP, Nakai H, Pandey K, Huang Z, Salazar FH, Xu H et al. Inhibition of hepatitis B virus in mice by RNA interference. Nat Biotechnol 2003; 21: 639-644.

23 Wu HL, Huang LR, Huang CC, Lai HL, Liu CJ, Huang YT et al. RNA interference-mediated control of hepatitis B virus and emergence of resistant mutant. Gastroenterology 2005; 128: 708-716.

24 Strayer DS, Feitelson M, Sun B, Matskevich AA. Paradigms for conditional expression of RNA interference molecules for use against viral targets. Methods Enzymol 2005; 392: 227-241.

25 Jacque JM, Triques K, Stevenson M. Modulation of HIV-1 replication by RNA interference. Nature 2002; 418: 435-438.

26 Lee NS, Dohjima T, Bauer G, Li H, Li MJ, Ehsani A et al. Expression of small interfering RNAs targeted against HIV-1 rev transcripts in human cells. Nat Biotechnol 2002; 20: 500-505.

27 Boden D, Pusch O, Lee F, Tucker L, Ramratnam B. Human immunodeficiency virus type 1 escape from RNA interference. J Virol 2003; 77: 11531-11535.

28 Li MJ, Bauer G, Michienzi A, Yee JK, Lee NS, Kim J et al. Inhibition of HIV-1 infection by lentiviral vectors expressing Pol III-promoted anti-HIV RNAs. Mol Ther 2003; 8: 196-206.

29 Das AT, Brummelkamp TR, Westerhout EM, Vink M, Madiredjo $\mathrm{M}$, Bernards $\mathrm{R}$ et al. Human immunodeficiency virus type 1 escapes from RNA interference-mediated inhibition. J Virol 2004; 78: 2601-2605.

30 Unwalla HJ, Li MJ, Kim JD, Li HT, Ehsani A, Alluin J et al. Negative feedback inhibition of HIV-1 by TAT-inducible expression of siRNA. Nat Biotechnol 2004; 22: 1573-1578.

31 Westerhout EM, Ooms M, Vink M, Das AT, Berkhout B. HIV-1 can escape from RNA interference by evolving an alternative structure in its RNA genome. Nucl Acids Res 2005; 33: 796-804.

32 Komano J, Miyauchi K, Matsuda Z, Yamamoto N. Inhibiting the Arp2/3 complex limits infection of both intracellular mature vaccinia virus and primate lentiviruses. Mol Biol Cell 2004; 15 : 5197-5207. 
33 Modem S, Badri KR, Holland TC, Reddy TR. Sam68 is absolutely required for Rev function and HIV-1 production. Nucl Acids Res 2005; 33: 873-879.

34 Plotkin SA, Orenstein WA, Offit PA. Vaccines. W.B. Saunders Company: Philadelphia, 2003.

35 Bitko V, Musiyenko A, Shulyayeva O, Barik S. Inhibition of respiratory viruses by nasally administered siRNA. Nat Med 2005; 11: 50-55.

36 Abbas-Terki T, Blanco-Bose W, Deglon N, Pralong W, Aebischer P. Lentiviral-mediated RNA interference. Hum Gene Ther 2002; 13: 2197-2201.

37 Verma IM, Weitzman MD. Gene therapy: twenty-first century medicine. Annu Rev Biochem 2005; 74: 711-738.

38 Judge AD, Sood V, Shaw JR, Fang D, McClintock K, Maclachlan I. Sequence-dependent stimulation of the mammalian innate immune response by synthetic siRNA. Nat Biotechnol 2005; 23: 457-462.

39 Jackson AL, Bartz SR, Schelter J, Kobayashi SV, Burchard J, Mao $\mathrm{M}$ et al. Expression profiling reveals off-target gene regulation by RNAi. Nat Biotechnol 2003; 21: 635-637.

40 Scacheri PC, Rozenblatt-Rosen O, Caplen NJ, Wolfsberg TG, Umayam L, Lee JC et al. Short interfering RNAs can induce unexpected and divergent changes in the levels of untargeted proteins in mammalian cells. Proc Natl Acad Sci USA 2004; 101: 1892-1897.

41 Yi R, Doehle BP, Qin Y, Macara IG, Cullen BR. Overexpression of exportin 5 enhances RNA interference mediated by short hairpin RNAs and microRNAs. RNA 2005; 11: 220-226.

42 Grimm D, Streetz KL, Pande K, Storm TA, Kay MA. Determinants of toxicity induced by in vivo expression of short hairpin RNA from AAV vectors. Mol Ther 2005; 11: S36-S36.

43 Nykanen A, Haley B, Zamore PD. ATP requirements and small interfering RNA structure in the RNA interference pathway. Cell 2001; 107: 309-321.

44 Xia H, Mao Q, Paulson HL, Davidson BL. siRNA-mediated gene silencing in vitro and in vivo. Nat Biotechnol 2002; 20: 1006-1010.

45 Song E, Zhu P, Lee SK, Chowdhury D, Kussman S, Dykxhoorn $\mathrm{DM}$ et al. Antibody mediated in vivo delivery of small interfering RNAs via cell-surface receptors. Nat Biotechnol 2005; 23: 709-717.

46 Chang Y, Chang SS, Lee HH, Doong SL, Takada K, Tsai CH. Inhibition of the Epstein-Barr virus lytic cycle by Zta-targeted RNA interference. J Gen Virol 2004; 85: 1371-1379.

47 Chen W, Yan W, Du Q, Fei L, Liu M, Ni Z et al. RNA interference targeting VP1 inhibits foot-and-mouth disease virus replication in BHK-21 cells and suckling mice. J Virol 2004; 78: 6900-6907.

48 Kahana R, Kuznetzova L, Rogel A, Shemesh M, Hai D, Yadin H et al. Inhibition of foot-and-mouth disease virus replication by small interfering RNA. J Gen Virol 2004; 85: 3213-3217.

49 Kapadia SB, Brideau-Andersen A, Chisari FV. Interference of hepatitis $C$ virus RNA replication by short interfering RNAs. Proc Natl Acad Sci USA 2003; 100: 2014-2018.

50 Kronke J, Kittler R, Buchholz F, Windisch MP, Pietschmann T, Bartenschlager $\mathrm{R}$ et al. Alternative approaches for efficient inhibition of hepatitis C virus RNA replication by small interfering RNAs. J Virol 2004; 78: 3436-3446.

51 Wilson JA, Richardson CD. Hepatitis C virus replicons escape RNA interference induced by a short interfering RNA directed against the NS5b coding region. J Virol 2005; 79: 7050-7058.

52 Novina CD, Murray MF, Dykxhoorn DM, Beresford PJ, Riess J, Lee SK et al. siRNA-directed inhibition of HIV-1 infection. Nat Med 2002; 8: 681-686.

53 Hall AH, Alexander KA. RNA interference of human papillomavirus type 18 E6 and E7 induces senescence in HeLa cells. J Virol 2003; 77: 6066-6069.

54 Butz K, Ristriani T, Hengstermann A, Denk C, Scheffner M, Hoppe-Seyler F. siRNA targeting of the viral E6 oncogene efficiently kills human papillomavirus-positive cancer cells. Oncogene 2003; 22: 5938-5945.
55 McCown M, Diamond MS, Pekosz A. The utility of siRNA transcripts produced by RNA polymerase $i$ in down regulating viral gene expression and replication of negative- and positivestrand RNA viruses. Virology 2003; 313: 514-524.

56 Fowler T, Bamberg S, Moller P, Klenk HD, Meyer TF, Becker S et al. Inhibition of Marburg virus protein expression and viral release by RNA interference. J Gen Virol 2005; 86: 1181-1188.

57 Barik S. Control of nonsegmented negative-strand RNA virus replication by siRNA. Virus Res 2004; 102: 27-35.

58 Gitlin L, Karelsky S, Andino R. Short interfering RNA confers intracellular antiviral immunity in human cells. Nature 2002; 418: 430-434.

59 Gitlin L, Stone JK, Andino R. Poliovirus escape from RNA interference: short interfering RNA-target recognition and implications for therapeutic approaches. J Virol 2005; 79: 1027-1035.

60 Dector MA, Romero P, Lopez S, Arias CF. Rotavirus gene silencing by small interfering RNAs. EMBO Rep 2002; 3: $1175-1180$

61 Arias CF, Dector MA, Segovia L, Lopez T, Camacho M, Isa P et al. RNA silencing of rotavirus gene expression. Virus Res 2004; 102: 43-51.

62 Bitko V, Barik S. Phenotypic silencing of cytoplasmic genes using sequence-specific double-stranded short interfering RNA and its application in the reverse genetics of wild type negative-strand RNA viruses. BMC Microbiol 2001; 1: 34.

$63 \mathrm{Li} \mathrm{T}$, Zhang Y, Fu L, Yu C, Li X, Li Y et al. siRNA targeting the leader sequence of SARS-CoV inhibits virus replication. Gene Therapy 2005; 12: 751-761.

64 Zhao P, Qin ZL, Ke JS, Lu Y, Liu M, Pan W et al. Small interfering RNA inhibits SARS-CoV nucleocapsid gene expression in cultured cells and mouse muscles. FEBS Lett 2005; 579: 2404-2410

65 Schwarz DS, Hutvagner G, Du T, Xu Z, Aronin N, Zamore PD. Asymmetry in the assembly of the RNAi enzyme complex. Cell 2003; 115: 199-208.

66 Drake JW, Charlesworth B, Charlesworth D, Crow JF. Rates of spontaneous mutation. Genetics 1998; 148: 1667-1686.

67 Martinez MA, Gutierrez A, Armand-Ugon M, Blanco J, Parera $\mathrm{M}$, Gomez J et al. Suppression of chemokine receptor expression by RNA interference allows for inhibition of HIV-1 replication. AIDS 2002; 16: 2385-2390.

68 Ji J, Wernli M, Klimkait T, Erb P. Enhanced gene silencing by the application of multiple specific small interfering RNAs. FEBS Lett 2003; 552: 247-252.

69 Anderson J, Banerjea A, Akkina R. Bispecific short hairpin siRNA constructs targeted to CD4, CXCR4, and CCR5 confer HIV-1 resistance. Oligonucleotides 2003; 13: 303-312.

70 Liu R, Paxton WA, Choe S, Ceradini D, Martin SR, Horuk R et al. Homozygous defect in HIV-1 coreceptor accounts for resistance of some multiply-exposed individuals to HIV-1 infection. Cell 1996; 86: 367-377.

71 Kameoka M, Nukuzuma S, Itaya A, Tanaka Y, Ota K, Ikuta K et al. RNA interference directed against Poly(ADP-Ribose) polymerase 1 efficiently suppresses human immunodeficiency virus type 1 replication in human cells. J Virol 2004; 78: 8931-8934

72 Chiu YL, Cao H, Jacque JM, Stevenson M, Rana TM. Inhibition of human immunodeficiency virus type 1 replication by RNA interference directed against human transcription elongation factor P-TEFb (CDK9/CyclinT1). J Virol 2004; 78: 2517-2529.

73 Yu Z, Sanchez-Velar N, Catrina IE, Kittler EL, Udofia EB, Zapp ML. The cellular HIV-1 Rev cofactor hRIP is required for viral replication. Proc Natl Acad Sci USA 2005; 102: 4027-4032.

74 Koga H, Ohshima T, Shimotohno K. Enhanced activation of taxdependent transcription of human T-cell leukemia virus type I (HTLV-I) long terminal repeat by TORC3. I Biol Chem 2004; 279: 52978-52983. 
75 Zhang J, Yamada O, Sakamoto T, Yoshida H, Iwai T, Matsushita $\mathrm{Y}$ et al. Down-regulation of viral replication by adenoviralmediated expression of siRNA against cellular cofactors for hepatitis C virus. Virology 2004; 320: 135-143.

76 Joost Haasnoot PC, Cupac D, Berkhout B. Inhibition of virus replication by RNA interference. J Biomed Sci 2003; 10: 607-616.

77 Durant J, Clevenbergh P, Halfon P, Delgiudice P, Porsin S, Simonet $\mathrm{P}$ et al. Drug-resistance genotyping in HIV-1 therapy: the VIRADAPT randomised controlled trial. Lancet 1999; 353: 2195-2199.

78 Leonard JN, Schaffer DV. Computational design of antiviral RNA interference strategies that resist human immunodeficiency virus escape. J Virol 2005; 79: 1645-1654.

79 Weinberger LS, Burnett JC, Toettcher JE, Arkin AP, Schaffer DV. Stochastic gene expression in a lentiviral feedback Loop: HIV-1
Tat expression drives phenotypic diversity. Cell 2005; 122: 169-182.

80 Endy D, Yin J. Toward antiviral strategies that resist viral escape. Antimicrob Agents Chemother 2000; 44: 1097-1099.

81 Beerenwinkel N, Daumer M, Oette M, Korn K, Hoffmann D, Kaiser $\mathrm{R}$ et al. Geno2pheno: estimating phenotypic drug resistance from HIV-1 genotypes. Nucl Acids Res 2003; 31: 3850-3855.

82 Beerenwinkel N, Lengauer T, Daumer M, Kaiser R, Walter H, Korn $\mathrm{K}$ et al. Methods for optimizing antiviral combination therapies. Bioinformatics 2003; 19 (Suppl 1): i16-i25.

83 Rambaut A, Posada D, Crandall KA, Holmes EC. The causes and consequences of HIV evolution. Nat Rev Genet 2004; 5: 52-61.

84 ter Hofstede HJ, Burger DM, Koopmans PP. Antiretroviral therapy in HIV patients: aspects of metabolic complications and mitochondrial toxicity. Neth J Med 2003; 61: 393-403. 